\title{
Composição das comunidades de planárias terrestres (Platyhelminthes, Tricladida, Terricola) do Parque Nacional dos Aparados da Serra, Brasil
}

\author{
Vanessa A. Baptista ${ }^{1,2}$, Lisiane B. Matos ${ }^{1,2}$, Israel A. Fick ${ }^{1,3}$ \& Ana M. Leal-Zanchet ${ }^{1,4}$ \\ 1. Instituto de Pesquisas de Planárias e Programa de Pós-Graduação em Biologia, Universidade do Vale do Rio dos Sinos (UNISINOS), \\ Av. Unisinos, 950, 93022-000 São Leopoldo, RS, Brasil. (zanchet@unisinos.br) \\ 2. Bolsista de iniciação científica do CNPq. \\ 3. Bolsista de Mestrado da CAPES. \\ 4. Pesquisador do CNPq.
}

\begin{abstract}
Community composition of land planarians (Platyhelminthes, Tricladida, Terricola) of the National Park of Aparados da Serra, Brazil. The National Park of Aparados da Serra (PNAS), located at the Eastern border of Araucarian Plateau, is one of the main protected areas of Southern Brazil, being characterized by the occurrence of fragments of mixed ombrophilous forest, surrounded by wet and dry grassland fields, and a continuous area of dense ombrophilous forest. We registered for the PNAS 35 species of land planarians, distributed into five genera (Geoplana Stimpson, 1857, Choeradoplana Graff, 1896, Notogynaphallia Ogren \& Kawakatsu, 1990, Pasipha Ogren \& Kawakatsu, 1990 and Cephaloflexa Carbayo \& Leal-Zanchet, 2003) of the family Geoplanidae, subfamily Geoplaninae. We observed 23 and 21 species, respectively, in the areas of mixed ombrophilous forest and dense ombrophilous forest. The Jaccard's similarity coefficient between the two areas was 0.42 . In areas of dry grassland field, only one species was observed. Seven species were collected in disturbed open habitats, four of them also observed in the forest areas. The known distribution of four species, i.e., Cephaloflexa bergi (Graff, 1899), Notogynaphallia graffi Leal-Zanchet \& Froehlich, 2006, Geoplana franciscana Leal-Zanchet \& Carbayo, 2001, and Geoplana josefi Carbayo \& Leal-Zanchet, 2001, the two latter ones having been known only from their type-locality, is amplified.
\end{abstract}

KEYWORDS. Survey, biodiversity, Terricola, Geoplanidae, Atlantic Forest.

RESUMO. O Parque Nacional dos Aparados da Serra (PNAS), localizado na borda oriental do Planalto das Araucárias, é uma das principais unidades de conservação do sul do Brasil, caracterizando-se pela ocorrência de fragmentos de floresta ombrófila mista, intermediada por campos secos e úmidos, e por uma área contínua de floresta ombrófila densa. Registraram-se, para o PNAS, 35 espécies de planárias terrestres, distribuídas em cinco gêneros (Geoplana Stimpson, 1857, Choeradoplana Graff, 1896, Notogynaphallia Ogren \& Kawakatsu, 1990, Pasipha Ogren \& Kawakatsu, 1990 e Cephaloflexa Carbayo \& Leal-Zanchet, 2003), pertencentes à família Geoplanidae, subfamília Geoplaninae. Observaram-se 23 e 21 espécies, respectivamente, nas áreas de floresta ombrófila mista e floresta ombrófila densa. O coeficiente de similaridade de Jaccard entre as duas formações foi de 0,42 . Em áreas de campo nativo foi observada apenas uma espécie. Sete espécies foram coletadas em áreas abertas antropizadas, sendo quatro delas também observadas nas áreas de floresta. Amplia-se a distribuição de quatro espécies, a saber, Cephaloflexa bergi (Graff, 1899), Notogynaphallia graffi LealZanchet \& Froehlich, 2006, Geoplana franciscana Leal-Zanchet \& Carbayo, 2001, e Geoplana josefi Carbayo \& Leal-Zanchet, 2001, as duas últimas conhecidas somente da sua localidade-tipo.

PALAVRAS-CHAVE. Levantamento, biodiversidade, Terricola, Geoplanidae, Floresta Atlântica.

A maioria dos estudos realizados no Brasil sobre a fauna de planárias terrestres foi desenvolvida com base em material de coletas esporádicas realizadas nos estados de São Paulo, Rio de Janeiro e Santa Catarina (OGRen \& KAWAKATSU, 1990). Assim, a maior riqueza de espécies de Terricola tem sido registrada para áreas cuja vegetação original era floresta ombrófila densa (SLuYs, 1998). No entanto, estudos recentes sobre a fauna de Terricola (Platyhelminthes) realizados na região do Planalto das Araucárias, no nordeste do Rio Grande do Sul, principalmente em áreas de floresta ombrófila mista, têm indicado a ocorrência de um grande número de espécies, várias delas novas para a ciência e/ou endêmicas para a região (CARBAYo et al., 2001, 2002; LEAL-ZANCHET \& Carbayo, 2000, 2001; Carbayo \& Leal-Zanchet, 2001, 2003; Froehlich \& LeAl-ZanChet, 2003).

Visando ampliar o conhecimento sobre a diversidade de planárias terrestres da região do Planalto das Araucárias, bem como comparar a composição faunística deste grupo em duas formações florestais distintas (floresta ombrófila mista e floresta ombrófila densa), desenvolveu-se o presente estudo no Parque Nacional dos Aparados da Serra. Esta área é contínua com o Parque Nacional da Serra Geral, totalizando cerca de 27.000 ha, representando uma das áreas de preservação mais importantes do sul do Brasil.

\section{MATERIAL E MÉTODOS}

O Parque Nacional dos Aparados da Serra (PNAS) localiza-se na borda oriental do Planalto das Araucárias $\left(29^{\circ} 05^{\prime} \mathrm{S}, 50^{\circ} 00^{\prime \prime} \mathrm{W}-29^{\circ} 15^{\prime} \mathrm{S}, 50^{\circ} 15^{\prime} \mathrm{W}\right)$ e apresenta área de 10.250 ha (Fig. 1). O PNAS possui fisionomias vegetais características, compostas por floresta ombrófila mista, com Araucaria angustifolia (Bert.) Kuntze, intermediada por campos secos e úmidos nas áreas de altitude elevada (cerca de $900 \mathrm{~m}$ ), no município de Cambará do Sul, Rio Grande do Sul, e por uma área contínua de floresta ombrófila densa, localizada a cerca de $40 \mathrm{~m}$ de altitude, em Santa Catarina. O clima da região é considerado 

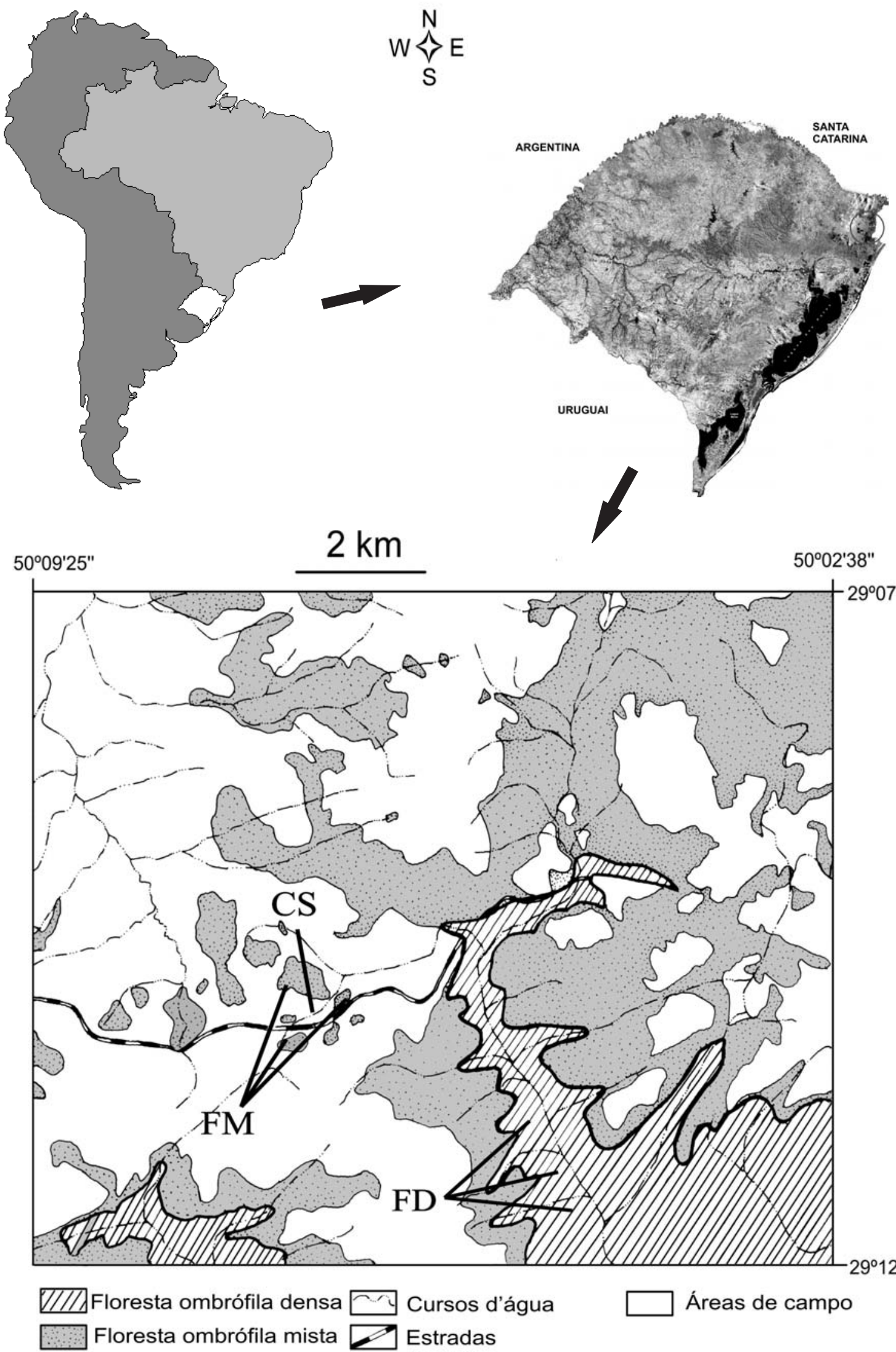

Áreas de campo

Fig. 1. Localização da área de estudo, Parque Nacional dos Aparados da Serra, Cambará do Sul, RS, e Praia Grande, SC, Brasil: pontos de coleta em áreas de campo seco (CS), floresta ombrófila densa (FD) e fragmentos de floresta ombrófila mista (FM). Adaptado de Fick et al. (2006). 
subtropical de acordo com Köppen (IBGE, 1986) e a precipitação anual varia de 1.500 a 2.250 mm (IBAMA, 2002).

O trabalho foi desenvolvido em três manchas de floresta ombrófila mista e em três locais da área contínua de floresta ombrófila densa (Fig. 1), perfazendo três repetições em cada tipo de hábitat. Além disso, foram investigadas duas áreas de campos secos e áreas abertas localizadas no entorno das construções do Parque. As coletas, diurnas, foram realizadas de forma direta, no folhiço e sob pedras, troncos e galhos caídos. Após as observações, o material era reposto na posição original para evitar alterações nos micro-hábitats, minimizandose, assim, possíveis impactos sobre a fauna (BALL \& REYNOLDSON, 1981; Winsor, 1997). De março de 2000 a março de 2002, foram feitas 24 observações nas áreas de floresta, com freqüência mensal. Para fins de análise quantitativa, foram demarcadas, aleatoriamente, parcelas de $7 \mathrm{~m} \times 7 \mathrm{~m}$, cuja posição era alterada mensalmente, sendo os animais procurados apenas no interior das parcelas (FicK et al., 2003, 2006). Complementarmente, para fins de levantamento faunístico, foram feitas observações em outros locais externos às parcelas. De abril de 2002 a março de 2003, foram realizadas três coletas adicionais não-aleatórias, isto é, sem demarcação de parcelas, selecionando-se, para observação, refúgios onde havia maior probabilidade de encontrar exemplares de planárias. Nas áreas de campo, o esforço de coleta foi menor, tendo sido efetuadas 16 observações, duas por estação. Adicionalmente, foram realizadas observações em bromélias epífitas localizadas nas duas formações florestais. $\mathrm{O}$ acesso às bromélias foi efetuado com o auxílio de corda para escalar os pontos mais altos; as folhas mais externas das plantas eram afastadas, efetuando-se, com auxílio de pincel, uma varredura nas mesmas, sem danificá-las. Foram analisadas 180 bromélias entre 1,5 m e $4 \mathrm{~m}$ de altura nas manchas de floresta ombrófila mista e 55 bromélias na área de floresta ombrófila densa.

Durante as atividades de coleta, os espécimes eram identificados em morfoespécies, de acordo com o padrão de coloração. Adicionalmente, os animais coletados foram analisados em laboratório quanto à forma do corpo, ao padrão de coloração, à distribuição dos olhos e à posição da boca e do gonóporo (Seitenfus \& LeAL-ZANChet, 2004). Para confirmação da identificação em nível de espécie, no mínimo dois exemplares de cada morfoespécie foram processados histologicamente, conforme metodologia descrita em CARBAYO \& LEAL-ZANCHET (2001). Espécimes incompletamente maduros foram identificados em nível de família, tendo sido considerados como táxons distintos somente aqueles que puderam ser diferenciados dos demais pela combinação de caracteres externos apresentada.

A similaridade entre as áreas de floresta foi estimada através do coeficiente de Jaccard (KREBS, 1989).

\section{RESULTADOS E DISCUSSÃO}

No Parque Nacional dos Aparados da Serra, registrou-se a ocorrência de 35 espécies de planárias terrestres (Tab. I), distribuídas em cinco gêneros: Geoplana Stimpson, 1857, Choeradoplana Graff, 1896, Notogynaphallia Ogren \& Kawakatsu, 1990, Pasipha
Ogren \& Kawakatsu, 1990 e Cephaloflexa Carbayo \& Leal-Zanchet, 2003, todos pertencentes à família Geoplanidae, subfamília Geoplaninae. Geoplana apresentou a maior riqueza de espécies, seguido por Pasipha e Notogynaphallia (Tab. I). Seis táxons foram identificados apenas até família, pois os espécimes coletados eram imaturos. No período de amostragem, 23 e 21 espécies, respectivamente, foram observadas em áreas de floresta ombrófila mista e densa. Houve seleção de hábitat por algumas espécies: 10 ocorreram exclusivamente nas áreas de floresta ombrófila mista e oito somente em áreas de floresta ombrófila densa. Treze espécies foram comuns às duas áreas (Tab. I). O coeficiente de similaridade de Jaccard entre as duas formações foi de 0,42 .

$\mathrm{Na}$ área de campo foi encontrado apenas um exemplar de Pasipha sp. 2, sob armadilha de pitfall, utilizada numa outra investigação. Quatro outras espécies tiveram registro exclusivo em áreas antropizadas, próximo a uma estrada (Geoplana sp. 9) ou no entorno das habitações do parque (Geoplana sp. 14, Geoplana sp. 15 e Pasipha sp. 4). Quatro espécies, Choeradoplana iheringi Graff, 1899, Geoplana franciscana Leal-Zanchet \& Carbayo, 2001, Geoplana sp. 5 e Pasipha sp. 2, foram registradas tanto nas áreas de floresta, como no entorno das habitações. Esses resultados indicam que estas espécies são mais generalistas quanto ao uso e à tolerância de hábitat. Choeradoplana iheringi já havia sido apontada por CARBAYO et al. (2002) como espécie indicadora de hábitats antropizados. No entanto, quanto a G. franciscana, os resultados aqui apresentados são discordantes daqueles obtidos por CARBAYO et al. (2002) para a Floresta Nacional de São Francisco de Paula, RS (FLONA/SFP), onde esta espécie havia sido sugerida como indicadora de hábitats preservados.

O esforço para amostragem na área de campo foi menor do que o empregado nas áreas de floresta, mas esperava-se obter um maior sucesso de captura com a realização de 16 amostragens nesse tipo de hábitat. O insucesso na obtenção de exemplares pode estar sendo influenciado pela quantidade muito grande de Poaceae, dificultando a coleta direta, e pela existência de poucos refúgios na área de campo. É provável que esse tipo de hábitat seja utilizado pelas planárias terrestres apenas para deslocarem-se de uma área de floresta para outra, pois sua sobrevivência por longos períodos em locais secos e ensolarados é dificultada pelo fato desses animais não possuírem mecanismos contra a perda d'água (Kawaguti, 1932).

Não foram encontradas planárias terrestres nas 235 bromélias observadas. Entretanto, foi observado, num dia com alta umidade relativa do ar, um exemplar de Notogynaphallia graffi Leal-Zanchet \& Froehlich, 2006 locomovendo-se no tronco de uma árvore viva (Myrtaceae), a 1,50 $\mathrm{m}$ de altura.

Ao comparar a estrutura das comunidades de planárias terrestres em áreas de floresta ombrófila mista e densa do PNAS, Fick et al. $(2003,2006)$ observaram 19 espécies. No presente trabalho, a maior duração da amostragem, a ampliação dos pontos de amostragem incluindo áreas abertas e a combinação de métodos de amostragem aleatórios e não-aleatórios resultaram numa 
maior riqueza observada (35 espécies). Adicionalmente, a área de ocorrência de algumas espécies, incluindo $G$. franciscana e Notogynaphallia graffi, registradas anteriormente somente em um dos dois tipos de floresta ombrófila do PNAS (Fick et al., 2003, 2006), foi ampliada para os dois tipos de floresta ombrófila, com os resultados do presente trabalho. Para fins de levantamento faunístico, constata-se que a utilização de métodos não-aleatórios aumenta as chances de localizar um maior número de animais, visto que os espécimes são procurados preferencialmente em pontos com maior umidade, onde há maior probabilidade de ocorrência de planárias.

A riqueza total registrada no PNAS, acima especificada, é similar à verificada na FLONA/SFP (40 espécies) por LEAL-ZANCHET \& CARBAYO (2000). No entanto, esperava-se para o PNAS uma riqueza maior, considerando que a área deste parque é cerca de cinco vezes maior que a da FLONA/SFP. Quanto à similaridade na composição das comunidades dessas duas unidades de conservação, verifica-se que cerca de dez espécies são comuns a ambas, uma baixa similaridade considerando a relativa proximidade entre as áreas $(65 \mathrm{~km})$ e a ocorrência de floresta ombrófila mista nas duas unidades. Dentre as espécies comuns ao PNAS e à FLONA/SFP, encontram-se Choeradoplana iheringi, Notogynaphallia graffi, Cephaloflexa bergi (Graff, 1899), Geoplana ladislavii Graff, 1899, G. josefi Carbayo \& Leal-Zanchet, 2001 e G. franciscana.
Choeradoplana iheringi e G. ladislavii têm distribuição relativamente ampla; a primeira havia sido registrada para os estados de Minas Gerais, Rio de Janeiro, São Paulo, Santa Catarina e Rio Grande do Sul (GrafF, 1899; RIESTER, 1938; MARCus, 1951; Froehlich, 1956a, 1959; LEAL-ZANCHET \& SouZA, 2003). Para G. ladislavii, há registros para Santa Catarina e Rio Grande do Sul (GrafF, 1899; FroeHLich, 1959), sendo ampla sua ocorrência neste último estado (CASTRO \& LeAL-ZANCHET, 2005). Para C. bergi, há registros do estado do Rio de Janeiro até Santa Catarina (GRAFF, 1899; RIESTER, 1938; Marcus, 1951; Froehlich, 1956a,b; Carbayo \& LealZANCHET, 2003), sendo este, portanto, o primeiro para o Rio Grande do Sul. Notogynaphallia graffi havia sido registrada anteriormente para a região nordeste do Rio Grande do Sul (LEAL-ZANCHET \& Froenlich , 2006). Geoplana franciscana e G. josefi tiveram registro anterior apenas para sua localidade-tipo, a FLONA/SFP(LEAL-ZANCHET \& CARBAYO, 2000, 2001; CARBAYo \& LeAL-ZANChET, 2001; CARBAYo et al. 2002), mas estudos recentes indicam sua ocorrência em outras áreas de floresta ombrófila mista do Planalto das Araucárias (A. M. Leal-Zanchet, obs. pess.). Neste estudo, é ampliada a distribuição de $N$. graffi, G. franciscana e $G$. josefi para o estado de Santa Catarina, em área de floresta ombrófila densa.

Algumas hipóteses podem ser levantadas para explicar a baixa similaridade das comunidades de planárias terrestres do PNAS e da FLONA/SFP, apesar da ocorrência

Tabela I. Tricladidos terrestres ocorrentes no Parque Nacional dos Aparados da Serra, Cambará do Sul, RS e Praia Grande, SC, Brasil (*, áreas de campo ou de entorno das habitações).

\begin{tabular}{|c|c|c|c|}
\hline Táxons & Floresta ombrófila mista & Floresta ombrófila densa & Outros * \\
\hline Geoplana ladislavii Graff, 1899 & - & $\mathrm{x}$ & - \\
\hline Geoplana franciscana Leal-Zanchet \& Carbayo, 2001 & $\mathrm{x}$ & $\mathrm{x}$ & $\mathrm{x}$ \\
\hline Geoplana josefi Carbayo \& Leal-Zanchet, 2001 & $\mathrm{x}$ & $\mathrm{x}$ & - \\
\hline Geoplana sp. 1 & $\mathrm{x}$ & $\mathrm{x}$ & - \\
\hline Geoplana sp. 2 & $\mathrm{x}$ & $\mathrm{x}$ & - \\
\hline Geoplana sp. 3 & $\mathrm{x}$ & $\mathrm{x}$ & - \\
\hline Geoplana sp. 4 & $\mathrm{x}$ & $\mathrm{x}$ & - \\
\hline Geoplana sp. 5 & $\mathrm{x}$ & $\mathrm{x}$ & $\mathrm{x}$ \\
\hline Geoplana sp. 6 & $\mathrm{x}$ & $\mathrm{x}$ & - \\
\hline Geoplana sp. 7 & $\mathrm{x}$ & - & - \\
\hline Geoplana sp. 8 & $\mathrm{x}$ & - & - \\
\hline Geoplana sp. 9 & - & - & $\mathrm{x}$ \\
\hline Geoplana sp. 10 & $\mathrm{x}$ & - & - \\
\hline Geoplana sp. 11 & - & $\mathrm{x}$ & - \\
\hline Geoplana sp. 12 & $\mathrm{x}$ & - & - \\
\hline Geoplana sp. 13 & $\mathrm{x}$ & - & - \\
\hline Geoplana sp. 14 & - & - & $\mathrm{x}$ \\
\hline Geoplana sp. 15 & - & - & $\mathrm{x}$ \\
\hline Geoplana sp. 16 & - & $\mathrm{x}$ & - \\
\hline Geoplana sp. 17 & - & $\mathrm{x}$ & - \\
\hline Cephaloflexa bergi (Graff, 1899) & $\mathrm{x}$ & - & - \\
\hline Choeradoplana iheringi Graff, 1899 & $\mathrm{x}$ & $\mathrm{x}$ & $\mathrm{x}$ \\
\hline Notogynaphallia graffi Leal-Zanchet \& Froehlich, 2006 & $\mathrm{x}$ & $\mathrm{x}$ & - \\
\hline Notogynaphallia sp. 1 & $\mathrm{x}$ & $\mathrm{x}$ & - \\
\hline Notogynaphallia sp. 2 & - & $\mathrm{x}$ & - \\
\hline Pasipha sp. 1 & - & $\mathrm{x}$ & - \\
\hline Pasipha sp. 2 & $\mathrm{x}$ & $\mathrm{x}$ & $\mathrm{x}$ \\
\hline Pasipha sp. 3 & - & $\mathrm{x}$ & - \\
\hline Pasipha sp. 4 & - & - & $\mathrm{x}$ \\
\hline Geoplanidae 1 & $\mathrm{x}$ & - & - \\
\hline Geoplanidae 2 & $\mathrm{x}$ & - & - \\
\hline Geoplanidae 3 & $\mathrm{x}$ & - & - \\
\hline Geoplanidae 4 & $\mathrm{x}$ & $\mathrm{x}$ & - \\
\hline Geoplanidae 5 & $\mathrm{x}$ & - & - \\
\hline Geoplanidae 6 & - & $\mathrm{x}$ & - \\
\hline
\end{tabular}


de floresta ombrófila mista em ambas. Essas áreas têm fitofisionomias distintas; na FLONA/SFP, as áreas de floresta ombrófila mista formam um mosaico juntamente com áreas de plantação de espécies nativas e exóticas, enquanto, no PNAS, são intermediadas por áreas de campo. Adicionalmente, diferentes tipos e níveis de impacto antrópico afetam as duas áreas. No PNAS, o impacto devese, nas áreas de maior altitude onde ocorre a floresta ombrófila mista, principalmente à presença de gado e a um processo de queimada anual nas áreas de campo ocorrentes no entorno do Parque. A área de floresta ombrófila densa apresenta indicativos de perturbação antrópica, como a presença de espécie exótica de roedor (FICK et al., 2006). Na FLONA/SFP, por sua vez, o maior impacto deve-se ao plantio de espécies exóticas (CARBAYO et al., 2002). Além disso, há outros fatores que podem estar contribuindo para a baixa similaridade entre as duas áreas, sendo importante destacar o fato de que comunidades biológicas possuem poucas espécies muito abundantes e algumas espécies de abundância média, sendo a maioria delas representada por poucos indivíduos (MAGURRAN, 1988). Estas últimas são mais difíceis de serem registradas, o que pode estar influenciando, em parte, os resultados da similaridade entre as duas áreas. A ampliação dos estudos sobre a composição de comunidades de planárias terrestres, inclusive com a comparação entre áreas de floresta ombrófila mista de diferentes locais, é necessária para uma melhor compreensão da distribuição das espécies nesse tipo de hábitat.

Agradecimentos. Ao CNPq e CAPES, pela bolsa de produtividade em pesquisa a A. M. Leal-Zanchet, bolsas de iniciação científica (PIBIC) e Mestrado aos demais autores. Ao Instituto Brasileiro do Meio Ambiente e dos Recursos Naturais Renováveis IBAMA, pela autorização para o desenvolvimento de pesquisas no Parque Nacional dos Aparados da Serra. Às laboratoristas Letícia A. Guterres e Aline F. Centa, pelo auxílio na confecção das preparações histológicas. À Profa. Dra. Eudóxia Froehlich, pelas sugestões realizadas numa versão preliminar do manuscrito.

\section{REFERÊNCIAS BIBLIOGRÁFICAS}

Ball, I. R. \& Reynoldson, T. B. 1981. British planarians. Cambridge, Cambridge University. 125p.

Carbayo, F. \& Leal-Zanchet, A. M. 2001. A new species of terrestrial planarian (Platyhelminthes, Tricladida: Terricola) from south Brazil. Brazilian Journal of Biology 61(3): $437-447$.

2003. Two new genera and species of Geoplaninae (Terricola: Tricladida: Platyhelminthes) of Brazil in the light of cephalic specialisations. Invertebrate Systematics 17(3):449-468.

Carbayo, F.; Leal-Zanchet, A. M. \& Vieira, E. M. 2001. Land planarians (Platyhelminthes: Tricladida: Terricola) as indicators of man-induced disturbance in a South Brazilian rainforest. Belgian Journal of Zoology 131(Suppl.):223-224.

2002. Terrestrial flatworm (Platyhelminthes: Tricladida: Terricola) diversity $v s$. man-induced disturbance in an ombrophilous forest from Southern Brazil. Biodiversity and Conservation 11:1091-1104.

Castro, R. A. \& Leal-Zanchet, A. M. 2005. Composição de comunidades de planárias terrestres (Platyhelminthes) em áreas de floresta estacional decidual e de campo na região central do Rio Grande do Sul, Brasil. Acta Biologica Leopoldensia 27(3):147-150.

Fick, I. A.; Leal-Zanchet, A. M. \& Vieira, E. M. 2003. Comparação da estrutura de comunidades de tricladidos terrestres
(Platyhelminthes: Terricola) em duas formações florestais do sul do Brasil. In: Congresso de ECologia do Brasil, 6 ${ }^{\circ}$, Fortaleza, 2003. Anais... Fortaleza, Universidade Federal do Ceará. p.152-153.

2006. Community structure of land flatworms (Platyhelminthes: Terricola): comparisons between Araucaria and Atlantic forest in Southern Brazil. Invertebrate Biology 125(4):306-313

Froenlich, C. G. 1956a. Tricladida Terricola das regiões de Teresópolis e Ubatuba. Papéis Avulsos do Departamento de Zoologia 12(16):313-344.

1956b. Planárias terrestres do Paraná. Dusenia 7(4):173-196. 1959. On geoplanids from Brazil. Boletim da Faculdade de Filosofia, Ciências e Letras da Universidade de São Paulo, Série Zoologia, 22:201-265.

Froehlich, E. M. \& Leal-Zanchet, A. M. 2003. A new species of terrestrial planarian of the genus Notogynaphallia Ogren \& Kawakatsu (Platyhelminthes, Tricladida, Terricola) from south Brazil and some comments on the genus. Revista Brasileira de Zoologia 20(4):745-753.

Graff, L. 1899. Monographie der Turbellarien: II. Tricladida Terricola. Leipzig, Engelmann. 574p.

IBAMA. 2002. Parque Nacional dos Aparados da Serra/ RS. Disponível em <http://www2.ibama.gov.br/unidades/ parques/reuc/65.htm>. Acesso em:11.04.2006.

IBGE. 1986. Levantamento de Recursos Naturais. Rio de Janeiro, SEPLAN/ Fundação Instituto Brasileiro de Geografia e Estatística - IBGE. v. 33, 796p.

KaWAGUTI, S. 1932. On the physiology of land planarians. III. The problems of desiccation. Memoirs of the Faculty of Science and Agriculture 7(1):39-55.

Krebs, C. J. 1989. Ecological methodology. New York, Harper \& Row. $654 \mathrm{p}$.

Leal-Zanchet, A. M. \& Carbayo, F. 2000. Fauna de planárias terrestres (Platyhelminthes, Tricladida, Terricola) da Floresta Nacional de São Francisco de Paula, RS, Brasil: uma análise preliminar. Acta Biologica Leopoldensia 22(1):19-25.

2001. Two new species of Geoplanidae (Platyhelminthes, Tricladida, Terricola) from Brazil. Journal of Zoology 253:433-446.

Leal-Zanchet, A. M. \& Froehlich, E. M. 2006. A species complex in the genus Notogynaphallia Ogren \& Kawakatsu (Platyhelminthes: Tricladida: Terricola) with a taxonomic revision of homonyms of Geoplana marginata Schultze \& Müller and a reinterpretation of Notogynaphallia caissara (Froehlich) anatomy. Belgian Journal of Zoology 136 (1):81-100.

Leal-Zanchet, A. M. \& Souza, S. A. 2003. Redescrição de Choeradoplana iheringi Graff (Platyhelminthes, Tricladida, Terricola). Revista Brasileira de Zoologia 20(3):523-530.

Magurran, A. 1988. Ecological diversity and its measurement. Princeton, Princeton University. 179p.

Marcus, E. 1951. Turbellaria brasileiros (9). Boletim da Faculdade de Filosofia, Ciências e Letras da Universidade de São Paulo, Série Zoologia, 16:5-215.

Ogren, R. E. \& Kawakatsu, M. 1990. Index to the species of the family Geoplanidae (Turbellaria, Tricladida, Terricola). Part I: Geoplaninae. Bulletin of the Fuji Women's College 28 Serie II:79-166.

Riester, A. 1938. Beiträge zur Geoplaniden-Fauna Brasiliens. Abhandlungen der senkenbergischen naturforschenden Gesellschaft 41:1-88.

Seitenfus, A. L. R. \& Leal-Zanchet, A. M. 2004. Uma introdução à morfologia e taxonomia de planárias terrestres (Platyhelminthes, Tricladida, Terricola). Acta Biologica Leopoldensia 26(2):187-202.

SLuYs, R. 1998. Land planarians (Platyhelminthes, Tricladida, Terricola) in biodiversity and conservation studies. Pedobiologia 42:490-494.

WinsoR, L. 1997. The biodiversity of terrestrial flatworms (Tricladida: Terricola) in Queensland: a preliminary report. Memoirs of Museum Victoria 56(2):575-579.

Recebido em dezembro de 2005. Aceito em maio de 2006. ISSN 0073-4721

Artigo disponível em: www.scielo.br/isz

Iheringia, Sér. Zool., Porto Alegre, 96(3):293-297, 30 de setembro de 2006 